\title{
Tricuspid Valve Infective Endocarditis In Drug Abusers : Clinical Features and Results of Surgical Treatment
}

\author{
Medvedev A.P.*, Lashmanov D.I.*, Bhandari Krishna*, Chiginev V.A.*, Pichugin V.V.*, \\ Zemskova E.N.*
}

* Nizhny Novgorod State Medical Academy; Cardiac and Vascular Surgery Center, Nizhny Novgorod, Russia

\section{ABSTRACT}

The objective of this study was to evaluate the clinical features, diagnostic criteria and indications for surgery in patients - drug abusers with tricuspid valve infective endocarditis (TVIE), and outcome of surgical treatment in these patients. From December 1999 to August 200935 patients (drug addicts) with TVIE were operated in the department of acquired heart diseases of Cardiac and Vascular Surgery Center, Nizhny Novgorod. 25 males and 10 females aged from 15 to 51 years were included in this study. 3 patients were re-operated due to recurrence of endocarditis. Biological prosthetic valve "Bio-Lab" was used in all patients. Intravenous drug abuse was the cause of the disease in all patients. Acute onset with hectic fever, shivering, profuse sweating, intoxication and development of multi-organ failure were the main clinical features of the disease. Embolism of the peripheral branches of pulmonary artery by septic embolus or micro thrombi were common symptoms. Ultrasound investigation played an important role in diagnosis of TVIE. It was the only criteria for the verification of the diagnosis in patients with fever of unknown origin until the appearance of valve damages and cardiac murmurs. All 35 (100\%) patients underwent tricuspid valve replacement (TVR). 3 (8.57\%) patients underwent redo TVR because of prosthetic valve endocarditis due to persistent intravenous drug abuse after surgery. The hospital mortality rate was $0 \%$. Acute debut of the disease may be the first clinical feature of tricuspid valve infective endocarditis in drug abusers. Ultrasound investigation is important for early diagnosis and effective treatment. Surgical treatment is indicated in cases of ineffective antibiotic therapy and massive tricuspid valve damages. Tricuspid valve replacement by a biological prosthesis was the treatment of choice in these patients. The use of biological prosthetic valve had good clinical results with low thrombogenic risk and high resistance to infection.

Key words: infective endocarditits, drug abusers, tricuspid valve replacement.

Correspondence:

Krishna Bhandari

Contacts: corsugeon@mail.ru;

Tel.: +79040424848;

Fax : $\quad+7(831)$ 4177790;

Postal Address: Vaneeva street, 209,

Nizhny Novgorod,

area code 603136 , Russia. 


\section{INTRODUCTION}

Isolated tricuspid valvular endocarditis is no longer rare; beginning from the 1970's, an increasing incidence in lesions of right chambers of the heart has been noted. In the 1990's, the rate of infective endocarditis in intravenous drug abusers (drug addicts) has progressively increased, exceeding $5-6 \%{ }^{1}, 33 \%$ of whom are younger than 25 years.

The affection of the right chambers of the heart by an infectious process is a characteristic complication in drug addicts, especially among intravenous drug abusers and is encountered in $60 \%$ of cases in isolation, in $14 \%$ - in association with a left-sided involvement (bilateral endocarditis), and in $26 \%$ only left chambers are involved ${ }^{2}$.

Infective endocarditis of tricuspid valve (TVIE) differs from left sided endocarditis, and is characterized by high resistance to antibiotic therapy, atypical clinical features, and severe pulmonary lesions due to embolic complications, toxic-septic liver lesions, severe intoxication and sepsis, early development of multi-organ failure (MOF), recurrence of infection.

Atypical clinical manifestations of TVIE cause late diagnosis, severe complications, and consequently inappropriate treatment. Even in confirmed cases, there is no standard treatment as this is a very new problem which has not been adequately elucidated in studies.

\section{OBJECTIVE}

The objective of this study was to evaluate the clinical features, diagnostic criteria and indications for surgery in patients - drug abusers - with tricuspid valve infective endocarditis, and outcome of surgical treatment in these patients.

\section{MATERIALS AND METHODS}

From December 1999 to August 200935 patients (drug addicts) with TVIE were operated in the department of acquired heart diseases of Cardiac and Vascular Surgery Center, Nizhny Novgorod. 25 males and 10 females aged from 15 to 51 years were included in this study. 3 patients were re-operated due to recurrence of endocarditis. The inclusion criteria for this study were: infec- tive endocarditis of tricuspid valve with narcomania.12 patients were excluded from this study as they had infective endocarditis of other valves : aortic valve (7); mitral valve (2); mitral and aortic valves (2) and tricuspid valve and valve of pulmonary artery (1). We analyzed clinical features of the disease, biochemical investigations, chest $x$-ray, ECG, ultrasound investigation and CT as required. Blood cultures were performed. All patients were operated with cardiopulmonary bypass and crystalloid cardioplegia. Biological prosthetic valve "Bio-Lab" ("BioLab" is a product of Bakulev scientific and research centre for cardiovascular surgery, Russian Academy of Medical Sciences, Moscow, Russia) was used in all patients. Morphological evaluation of the excised tricuspid valve was performed.

\section{RESULTS}

Intravenous drug abuse was the cause of the disease in all patients. Acute onset with hectic fever, shivering, profuse sweating, intoxication and development of multi-organ failure were the main clinical features of the disease. Embolism of the peripheral branches of pulmonary artery by septic embolus or micro thrombi was a common symptom. Clinical manifestations of the disease are presented in the table 1.

The majority of the patients (30 or $85.71 \%$ ) were in IV functional class NYHA and only 5 (14.28\%) were in III functional class NYHA.

Twenty four patients $(68.57 \%)$ had serious pulmonary complications as destructive pneumonia. Preoperative management of these patients was performed in pulmonary department. These patients were managed by physicians and cardiologists. Active effective antibiotic therapy reduced inflammatory changes in the lungs leading to improved respiratory function. Surgery was performed in patients unresponsive to medical therapy.

Blood culture was positive in 35 (100\%) patients, Staphylococcus aureus was found.

Ultrasound investigation played an important role in diagnosis of TVIE. It was often the only critria for verification of the diagnosis in patients with fever of unknown origin until the appearance of valve damages and cardiac murmurs. Ultrasound 
investigation is an important method to diagnose the vegetations in tricuspid valve cusps. Vegetations more than $3 \mathrm{~mm}$ can be revealed with ultrasound. According to some authors ${ }^{3,4}$, vegetations can be revealed by ultrasound only when duration of illness exceeds 6 weeks.
Careful and detailed analysis of all clinical features, medical reports, results of biochemical and instrumental investigations are necessary for accurate and on-time diagnosis of TVIE. Definitely, the use of bactericidal antibiotics is a basic method for treatment of IE. Antibiotic therapy for

Table 1 Clinical manifestations of tricuspid valve infective endocarditis in drug abusers

$\begin{array}{lcc}\text { Clinical Features } & \text { Number of patients } & \% \\ \text { Acute phase with the development of MOF } & 35 & 100 \\ \text { Embolism of pulmonary artery with pulmonary } & & \\ \text { infarction and abscess formation } & 24 & 68.57 \\ \text { Hepatitis C } & 25 & 71.42 \\ \text { Hepatitis B and C } & 5 & 14.28 \\ \text { Diffuse glomerulonephritis } & 19 & 54.28 \\ \text { Splenomegaly } & 25 & 71.42 \\ \text { ESR >60mm/hr } & 20 & 57.14 \\ \text { Exudative pericarditis } & 5 & 14.28 \\ \text { Pleuritis } & 6 & 17.14\end{array}$

Fig.1. ECHO, parasternal position with short axis: destruction of septal and anterior leaflets of TV, prolapsed fragments of leaflets (arrow) into RA (RA - right atrium; RV - right ventricle; $A O$ - aorta).

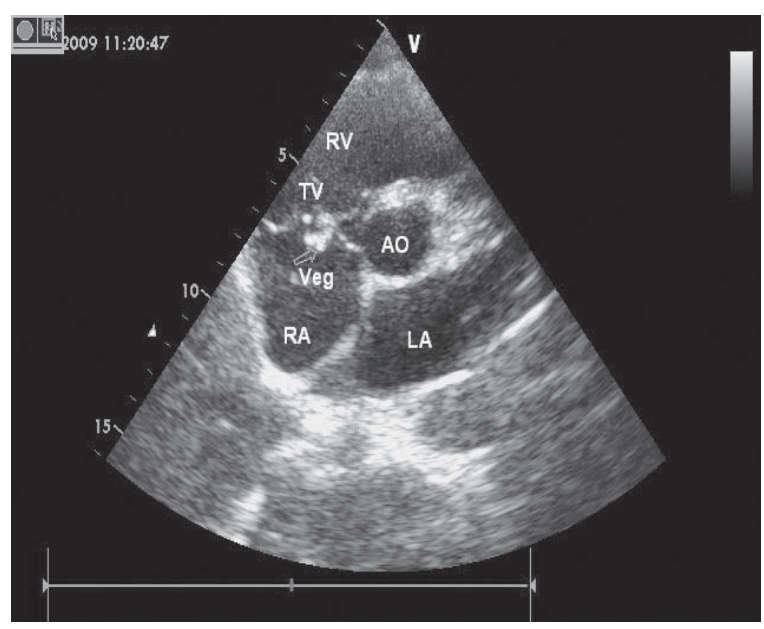

Fig.2. ECHO, apical four-chambered position massive vegetations (arrow) on tricuspid valve leaflets (RV - right ventricle; RA - right atrium; LV - left ventricle; LA - left atrium).

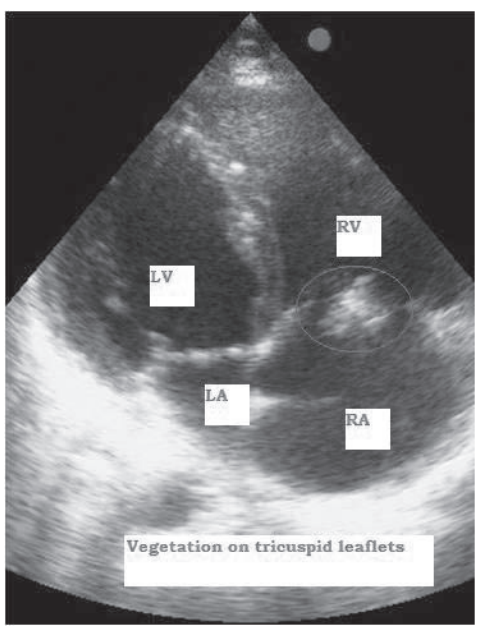

Table 2. Morphological changes of tricuspid valve in drug abusers

$\begin{array}{lcc}\text { Operative Findings } & \text { Number of patients } & \% \\ \text { Massive vegetations } & 35 & 100 \\ \text { Destruction, rupture, perforation of cusps } & 35 & 100 \\ \text { Cusps ulceration } & 35 & 100 \\ \text { Chordae detachment } & 35 & 100 \\ \text { Paravalvular abscesses } & 3 & 8.57 \\ \text { Parietal endocarditis } & 24 & 68.57\end{array}$


IE should be prolonged to get remission and to prevent relapse. Currently, early adequate and effective antibiotic therapy improves prognosis in IE. Unfortunately, this is not always possible in drug addicts because of difficulties in diagnosis, widespread microbial resistant strains and recurrence of the infective process. They have a high risk of severe damage of valvular structures with the development of heart failure, massive oscillating vegetations, pulmonary embolism, paravalvular spreading of infection, formation of abscesses and others changes. Surgical treatment is method of choice in these patients. The best way to solve this problem is development and improvement of surgical methods.

Indications for surgical treatment were as follows: 1 ) ineffectiveness of antibiotic therapy and presence of massive oscillating vegetations on damaged leaflets of tricuspid valve; 2 ) tricuspid valve regurgitation (3-rd degree) and 3) recurrent episodes of pulmonary embolism with the development of destructive pneumonia.

All $35(100 \%)$ patients underwent tricuspid valve replacement. $3(8.57 \%)$ patients underwent redo TVR because of prosthetic valve endocarditis due to persistent intravenous drug abuse after surgery. Intraoperative findings of changes of tricuspid valve are presented in the table 2 .

All patients were discharged from the clinic with uneventful postoperative recovery. The hospital mortality in our study group patients was $0 \%$.

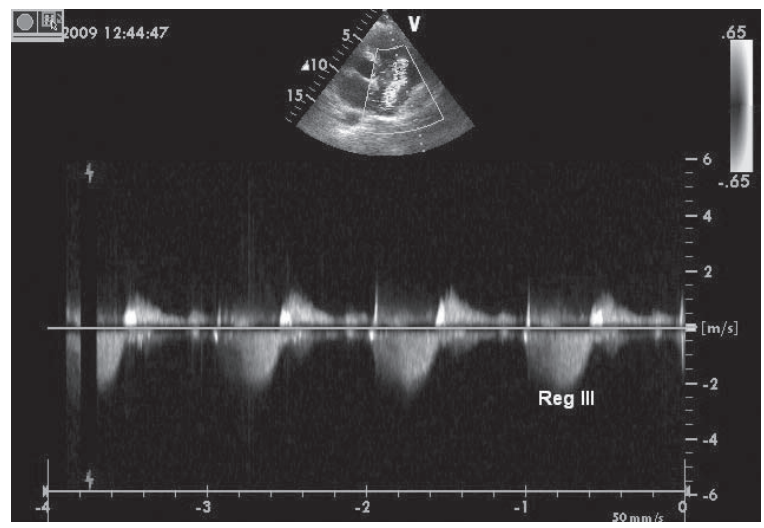

\section{DISCUSSION}

Due to the following features, IE among drug abusers is classified separately: atypical clinical features, pulmonary involvement with the development of pulmonary embolism, severe septic process and multi-organ failure, resistance to antibiotic therapy.

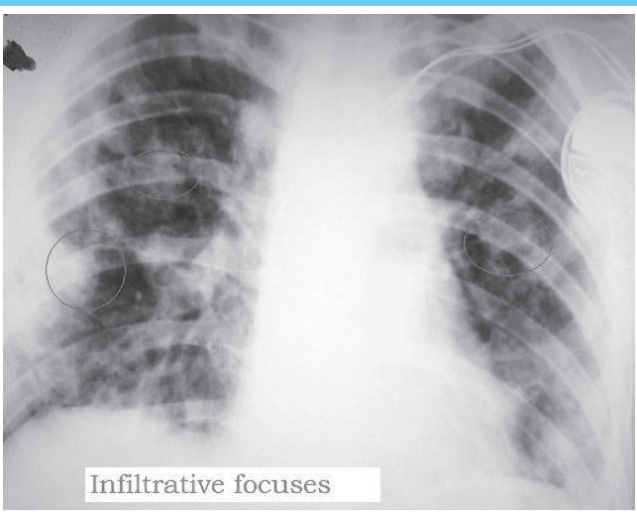

At present, the following are the indications for operative management of TVIE among drug abusers:

1. intractable infectious process;

2. recurrence of pulmonary artery embolism and its risk;

3. progressive circulatory failure.

The management of such patients is determined by response and duration of antibiotic therapy, adequate etiological treatment.

Timing and indication of surgery requires experienced clinical judgment and is decided by the cardiologist.

Identification of pathogenic agent is important to evaluate prognosis. Conservative management leads to mortality in patients with polyantibiotic resistant Staphylococcus aureus. Operation should be performed in ineffective antibiotic therapy and before development of pulmonary complications and valvular damage when S. aureus is isolated in blood culture.

The important clinical features among patients with TVIE are presence of pulmonary complications: septic pneumonia, infarction-pneumonia, multiple staphylococcal destruction. Severe pulmonary complication such as abscess with pronounced respiratory failure limits to operative management.

Surgery may be required when recurrent emboli are detected and medical treatment is ineffective. Surgical management of such patients gives sound results before the development of pulmonary embolism ${ }^{5}$. That's why to formulate indication to surgical management - risk of development of pulmonary embolism; ultrasound plays a determining role. Massive $(>\mathrm{I} \mathrm{cm})$ mobile 
vegetations may cause serious embolic complications. Appearance and potential embologenic vegetations need individual evaluation, but it is understood that operation should be performed before the embolic event.

In patients on inotropic support, with progressing cardiac failure surgical management should be considered. The progression of cardiac failure in patients with TVIE is not only due to destruction of TV but also due to toxic infectious and immune inflammatory damage of the myocardium. Early diagnosis and treatment of TVIE, minimizes the

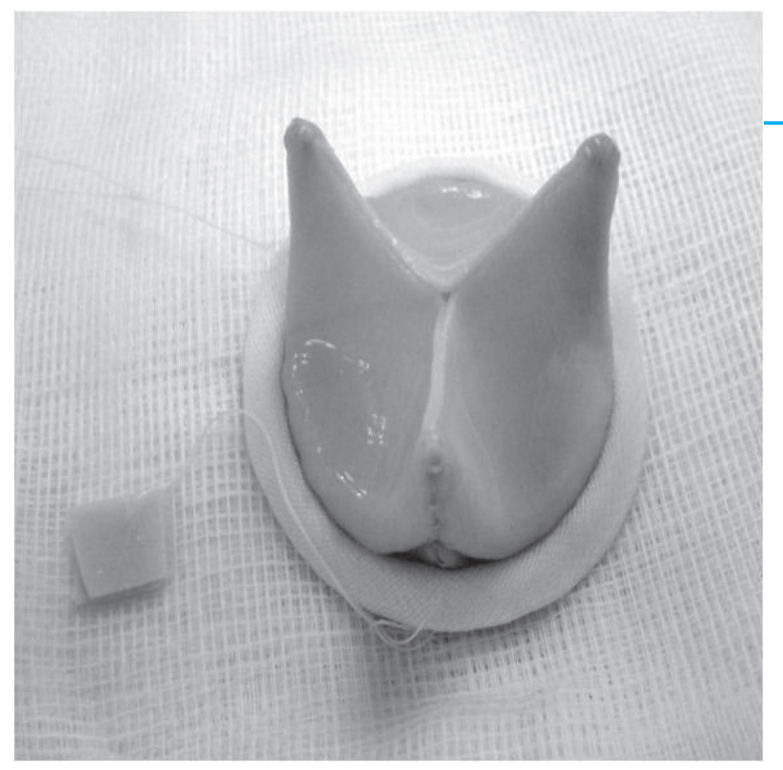

destruction of TV components and myocardium, thus allowing valve-sparing operations. Thus, severe heart failure is a late indication for surgery ${ }^{6}$.

There are few methods of surgical management of TVIE. Two-step operative management to the patients with septic clinical features is suggested. In first step, affected valve is excised and infective focus removed. During the second step valve replacement is performed 3-4 months after the sepsis has subsided 7,8 . Clinical studies have showed that two-step method can be used in critical patients with intractable septic process in the absence of pulmonary hypertension ${ }^{9,10}$. Supporter of this method - leads to high probability of reoperation - mortality rate after first step is $15 \%$ and after second step $25 \%$ due to long-standing severe tricuspid regurgitation ${ }^{11,12}$. We didn't use this type of surgical treatment. The surgical management of such patients includes cardiac chamber sanitation and tricuspid valve replacement. Valve replacement eliminates tricuspid valve regurgitation but risk of prosthetic endocarditis is a life-threatening complication in persistent intravenous drug abusers. Mostly redo operations are ineffective. That's why, it is concluded that medical management of prosthetic endocarditis is the treatment of choice among intravenous drug users.

There are many controversies regarding tricuspid valve replacement in intravenous drug abusers. Mechanical prosthetic valves are rarely used in tricuspiu position due to higiा risk of tiाrominosis associated with low velocity of blood flow in right heart chambers. The recommended range of INR is $3.5-4.0$ for mechanical prosthetic valve and this causes an increased risk of bleeding. In many countries access to INR control is limited. Biological prosthetic valves are more preferable in tricuspid position.

The use of biological prosthetic valve in tricuspid valve surgery considerably minimizes postoperative risk of thrombosis and free from oral anticoagulants. This is confirmed in our clinical study with biological prosthetic valve "Bio-Lab" in surgery for TVIE in intravenous drug abusers.

\section{CONCLUSIONS}

1. Acute debut of the disease may be the first clinical feature of tricuspid valve infective endocarditis in drug abusers.

2. The ultrasound investigation is important for early diagnosis and effective treatment.

3. Surgical treatment is indicated in cases of ineffective antibiotic therapy and massive tricuspid valve damages. Replacement of tricuspid valve by biological prosthesis was the method of choice for the patients with tricuspid valve infective endocarditis.

4. The use of biological prosthetic valve had good clinical results with low thrombogenic risk and high resistance to infection. 


\section{REFERENCES}

1. Demin A. A, Drobisheva V. P., Velter 0. Y. Infective endocarditis among intravenous drug abusers//Clinical medicine-2000-№8 p.4752.

2. Masters C. A., Saba J., Dassagangas J. et al. The $t$ surgical treatment of infective endocarditis in drug addicts//European Heart Journal-1987-vol.8-suppl.3, p.375-377.

3. Storojhilov G. I., Kasatova T. B., Butkevich O. M. Experience of ECHO use among patients with infective endocarditis//Therapeutic achieve-1980, v-52,№10, p.63-64.

4. Gonska B.D., sold G., Kreuzer H. Dedeutung des echocardiographishen Vegatationsnachweses fur den klinischen Verlauf und die Prognose bie infection Endokarditis//Ztschr. Kardiol.-1984-Bd.73-H.7-s.455-459.

5. Moisev V. S., Troyanova T.G., Milto A S. Infective endocarditis in drug addicts/ZClinical medicine-1998-№11, p.31-33.

6. Shevchenko Y.L., Khabulava G.G Infective endocarditis of right heart chambers//SaintPetersburg-Science-1996, $170 \mathrm{p}$.
7. Arbulu A., Thorns N.W., chiscan A. et al. Total tricuspid valvectomy without replacement in the treatment of Pseudomonas endocarditis// Surg.Forum.-1971, vol.22, p.163-164.

8. Chandraratna P.A.N., Reagan R.B., Imaizumi T. et al. Infective endocarditis cured by resection of a tricuspid valve vegetation//Ann. Intern. Med.-1978, vol.89, №4, p.517-518.

9. Turley K. Surgery of right-sided endocarditis valve preservation versus replacement //J. Card. Surg.-1989, vol.4, №4, p.317-320.

10. Louie E.K., bienizrz T., Moore A.M. et al. Reduced atrial contribution to left ventricular filling in patients with severe tricuspid valvulectomy: A Doppler echocardiographic study//J. Amer. Coll. Cardiol -1990, vol.16, №7, p.1617-1624.

11. Evora PR., Brasil J.C., Elias ML. et al. Surgical excision of the vegetation as treatment of tricuspid valve endocarditis//Cardiology -1988, vol.75, №4, p.287-288.

12. Arbulu A., Astaw J. Management of infective endocarditis: Seventeen years experience// Ann. Thorac. Surg.-1987, vol.43, №2, p.144149. 